\title{
Service Life Prediction of Masonry Arch Bridges Using Artificial Neural Networks
}

\author{
P. B. R. Dissanayake and S. B. Narasinghe
}

\begin{abstract}
This paper presents a methodology to predict reliability based remaining service lives and estimation of serviceability conditions of masonry arch bridges using Artificial Neural Networks (ANNs). In this ANNs analysis, training was processed by Back-Propagation (BP) Algorithm with corresponding parameters. The critical failure mode of the masonry arch bridge is based on axle loads. The parameters for Back-Propagation are mean value $\left(\mu_{M}\right)$ and standard deviation $\left(\sigma_{M}\right)$ of proposed safety margin of the masonry arch bridge. Those parameters were used to predict the serviceability condition of the masonry arch bridges. Finally, the remaining service life of the masonry arch bridge was determined using a target failure probability, while assuming that the current rate of loading magnitude and frequency are constant for future prediction. Proposed methodology is illustrated with a case study bridge selected from the national road network of Sri Lanka.
\end{abstract}

Keywords: Artificial Neural Networks, Back-Propagation, Reliability Index, FailureProbability, Service Life

\section{Introduction}

The masonry arch is one of the oldest forms of bridges. The first known ones were probably built in the Middle East around 5500 years ago. The masonry arch bridges were common in olden days because they were cheaper and simpler ways of spanning the gaps. In Sri Lanka, there are more than 100 arch bridges in the national road network. Most of those are either rock or brick arch bridges. The appearance of arch bridges in Sri Lanka began in the initial part of the colonial period [5]. Due to current road development programs in Sri Lanka, there is a tendency of replacing these old arch bridges with newer forms of wider and stronger bridges. With such assessment of these structures, future conservation and replacement can be carried out a proper way. Figure 1 shows the general configuration of a masonry arch bridge.

\section{Literature Review}

Back-propagation neural networks are adapted to training the data, as they have a high capability of data mapping. Back-propagation neural networks have been widely used as a learning algorithm in feed forward multilayer neural networks. It is applied to feed forward Artificial Neural Networks (ANNs) with one or more hidden layers. Also BP neural networks have been applied to a wide range of areas including classification, estimation, prediction and functions synthesis and they are currently the most widely used neural networks. What makes this algorithm different from the others is the process by which the weight is calculated during the learning phase of the networks.

An ANN model can be manipulated in many ways to improve its performance, including varying its internal architecture, learning paradigm or parameters, or modifying the data set used to "train" it. Given the large number of possible network configurations, choosing an appropriate network paradigm and architecture is still largely art rather than science [3]. Various configurations of numbers of processing elements and hidden layers are used in the Back-Propagation ANNs. BP is one paradigm

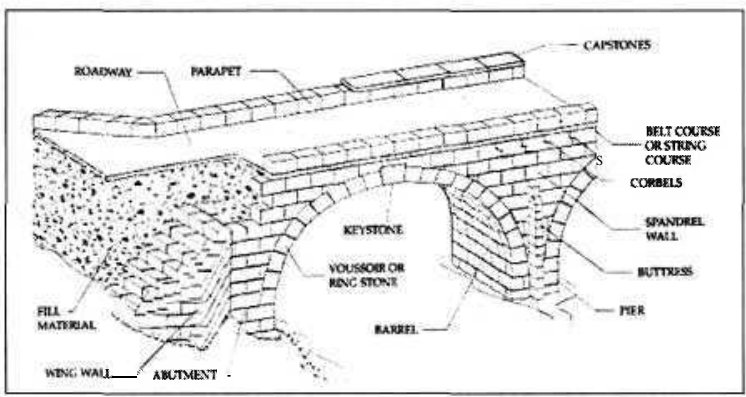

Figure 1 - Main parts of the masonry arch bridge

Eng. (Dr.) P.B.R. Dissanayake, C. Eng., MIE(SL),B.Sc. Eng. (Hons) (Peradeniya),M.Eng. (Ehime),Dr. Eng. (Ehime), Senior Lecturer in Civil Engineering, Department of Civil Engineering, Universityof Peradeniya. Eng. S. B. Narasinghe, B.Sc. Eng. (Hons) (Peradeniya),AMIESL. 
for network learning that involves changing connection weights between hidden units based on the contribution each has made towards generating an erroneous output during training. In addition to experimentation by trial and error with various network architectures, the cascade correlation algorithm for developing ANN architectures is used to guide the trial and error. In this algorithm, an ANNs model begins with a single hidden processing element and adds new neurons while iterating through the training set until performance is no longer improved by adding new hidden units. At this point, the architecture of the network can be considered to be optimal [3]. The ANN method has another advantage over the conventional methods in that once the model is trained; the model can be used as an accurate and quick tool for estimating the service lives of bridges.

\section{Preparation}

In performing reliability based analysis of masonry arch bridges, the first step is to derive a suitable reliability expression. For that it is essential to pay attention towards possible failure criterion. In this study, critical failure mode of masonry arch bridges is based on axel load on the bridge. Based on those foundations reliability expression or safety margin of the masonry arch bridges is as follows [6],

$M=\mathrm{PAL}-A A L$

Where $\mathrm{M}$ is the safety margin, PAL is the Provisional Axle Load in $k N s$ and AAL is the Actual Axle Load in $k N s$. Both axle loads are not deterministic quantities. In this regard, they are assumed to behave as random variable with some probabilistic parameters. PAL represents the strength variables whereas AAL represents the load variable in the reliability model introduced in Eq.1.

When, PAL $>$ AAL, M $>0$, the bridge is in safe region and When, $\mathrm{PAL}<\mathrm{AAL}, \mathrm{M}<0$, and bridge is in failure region.

If both random variables are normally distributed, Such that

$$
\begin{aligned}
& P A L \sim N\left(\mu_{P A L}, \sigma_{P A L}\right) \\
& A A L \sim N\left(\mu_{A A L}, \sigma_{A A L}\right)
\end{aligned}
$$

Then reliability index $(\beta)$ which is a measure of number of standard deviations $\left(\sigma_{M}\right)$ from mean value $\left(\mu_{M}\right)$ to the failure point, can be found as follows,

$$
P=\mu_{M} / \sigma_{M} \text {. }
$$

As $\mathrm{M}$ is a linearly independent combination of PAL and AAL

$$
\beta=\left(\mu_{P A L}-\mu_{A A L}\right) /\left(\sigma_{P A L}^{2}+\sigma_{A L L}^{2}\right)^{0.5}
$$

Failure probability $\mathrm{P}$, which is a measure of how close the failure of the element, at a selected time, can be expressed as,

$$
P_{f}=\mathrm{P}(\mathrm{M} \leq 0)
$$

Having converted $M$ in to the standard normally distributed variable, a relationship with failure probability with reliability index can be found as in Eq.2.

$P_{f}=\varnothing(-\beta)$

It is possible to estimate the elementary reliability indices as above.

In calculating PAL, Military Engineering experimental Establishments (MEXE) was used.

$$
P A L=740^{*}(d+h)^{2} / L^{1.3} \text { in } k N
$$

As a case study, a single spanned stone masonry arch bridge (71/1) situated close to Hatton town in A7 road in the national road network in Sri Lanka was selected. This bridge was constructed in 1918 and it has been in operation ever since. It has following geometric details.

$$
\begin{array}{lll}
\text { Clear Span of the arch }(\mathrm{L}) & = & 8.8 \mathrm{~m} \\
\text { Thickness of the barrel }(d) & = & 0.55 \mathrm{~m} \\
\begin{array}{l}
\text { Height of the compacted soil } \\
\text { at the crest }(h)
\end{array} & =1.5 \mathrm{~m}
\end{array}
$$

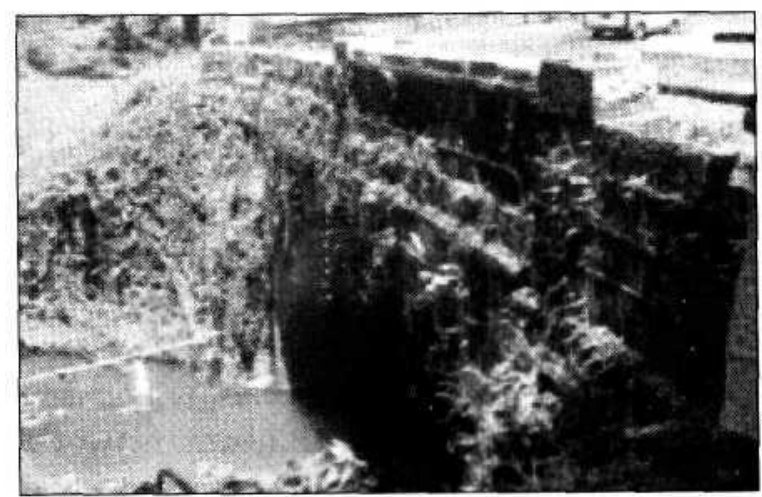

Figure 2 - Single spanned stone masonry arch bridge (71/1) situated close to Hatton town 
Initially, PAL is calculated using MEXE method (Eq.3.). That was $184.03 k N$. This initial estimated PAL should be corrected with five modification factors, such as span/rise, profile, material, joint and condition factors. Therefore, all adjustment factors are multiplied with the initial estimated PAL to get corrected value for PAL. Now with these adjustment factors and using the above method, modified value of PAL is $234.06 \mathrm{kN}$. From modified MEXE method, assuming Coefficient of Variation (COV) is 0.1 and calculates the Stranded Deviation $23.41 \mathrm{kN}$. From axel load measurements, it is found that AAL has a mean of $50.7 \mathrm{kN}$ and Standard deviation of $29.03 \mathrm{kN}$.

$A A L$ is obtained using weigh in motion measurements at the particular bridge site. For this purpose, a pneumatic tube was employed at the site. From the sample readings gathered over a period of week for a particular year of 2003, probabilistic parameters of the AAL can be found. Based on that, the probabilistic parameters of AAL from 1918 to 2003 were calculated and they are as shown in the Table 1 below.

Finally, based on the reliability based ANN model for the particular bridge at different

Table 1 - Basic parameter of the Reliability model

\begin{tabular}{|c|c|c|c|c|c|}
\hline \multirow{2}{*}{ Pattem } & \multirow{2}{*}{ Year } & \multicolumn{2}{|c|}{ PAL } & \multicolumn{2}{c|}{ AAL } \\
\cline { 3 - 6 } & & $\begin{array}{c}\text { COVAL } \\
\text { MPA }\end{array}$ & \multicolumn{1}{c|}{$\sigma$ PAL } & $\mu$ AAL & $\sigma$ AAL \\
& & $/(\mathrm{kN})$ & $/(\mathrm{kN})$ & $/(\mathrm{kN})$ \\
\hline 1 & 1918 & 246.38 & 24.63 & 10.14 & 24.01 \\
2 & 1923 & 245.66 & 24.57 & 11.41 & 11.41 \\
3 & 1928 & 244.93 & 24.49 & 12.68 & 12.68 \\
4 & 1933 & 244.21 & 24.42 & 13.94 & 13.94 \\
5 & 1938 & 243.48 & 24.35 & 15.21 & 15.21 \\
6 & 1943 & 242.76 & 24.27 & 16.48 & 16.48 \\
7 & 1948 & 242.04 & 24.2 & 17.75 & 17.75 \\
8 & 1953 & 241.31 & 24.13 & 19.01 & 19.01 \\
9 & 1958 & 240.59 & 24.06 & 20.28 & 20.28 \\
10 & 1963 & 239.86 & 23.99 & 21.55 & 21.55 \\
11 & 1968 & 239.14 & 23.91 & 22.81 & 22.82 \\
12 & 1973 & 238.41 & 23.84 & 24.08 & 24.08 \\
13 & 1978 & 237.69 & 23.77 & 25.35 & 27.58 \\
14 & 1983 & 236.96 & 23.7 & 30.42 & 27.87 \\
15 & 1988 & 236.24 & 23.62 & 35.49 & 28.16 \\
16 & 1993 & 235.51 & 23.55 & 40.56 & 28.44 \\
17 & 1998 & 234.79 & 23.48 & 45.63 & 28.74 \\
18 & 2003 & 234.06 & 23.41 & 50.7 & 29.03 \\
\hline
\end{tabular}

times, failure probabilities can be predicted and plotted with time. Then with the target failure probability of bridges set, the time to reach the current failure probability to the target probability can be found. This is the remaining service life of the selected bridge.

\section{Details}

\subsection{Neural Network model for Service life Prediction of Masonry Arch Bridge}

\subsubsection{Computer Program}

There are several software packages available to run the ANN with different facilities. For this research, JavaNNS developed by University of Tuvingen was used. This user-friendly graphical interface was written in Java computer language Here, while training the model error graph, weight changes in the nodes and performance of model can be observed separately.

\subsubsection{Model inputs}

An important step in developing the neural network model is to select the input variables that have significant influence on settlement. The following variables are selected as input variables for the Back-Propagation neural network:

$$
M_{P A L^{\prime}} \sigma_{P A L^{\prime}} \mu_{A A L} \text { and } \sigma_{A A L} .
$$

Depending on the type of ANN model selected, inputs to processing elements must typically be either between 0 and 1 , or -1 and 1 . For the purpose of this research, a valid data range of 0 to 1 . The Selected Reliability Index is the only output variable in the neural Network.

\subsubsection{Development of an ANN Model}

Using the JavaNNS software, a network with one hidden layer and 7 hidden neurons was found to perform best. Here 18 patterns (data set) were obtained to analysis the model. Then first 17 patterns were trained and one pattern (2003) selected as a validation pattern. By observing the Error graph, best-performed model was repeated by ANNs. 
The best performed model had following parameters;

- No. of inputs: 4

- Training Function: Backpropergation

- Learning Coefficient(c): 0.3

- Momentum (m): 0.4

- No. of hidden layers: 1

- No. of neurons in the hidden layer: 7

- No. of out put: 1

\subsection{Results}

The plots of the calculated and predicted Reliability Index and Failure Probabilities are shown in Figures 3 and 4 respectively.

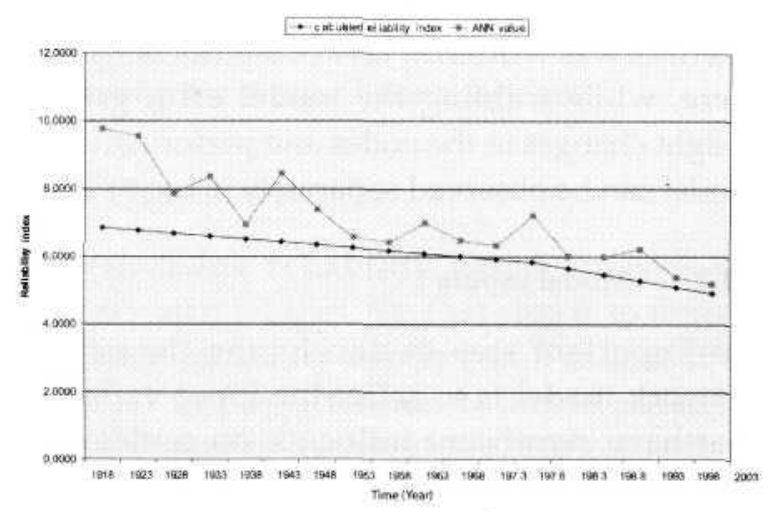

Figure 3 - Comparison of calculated and predicted Reliability Index for ANN training set

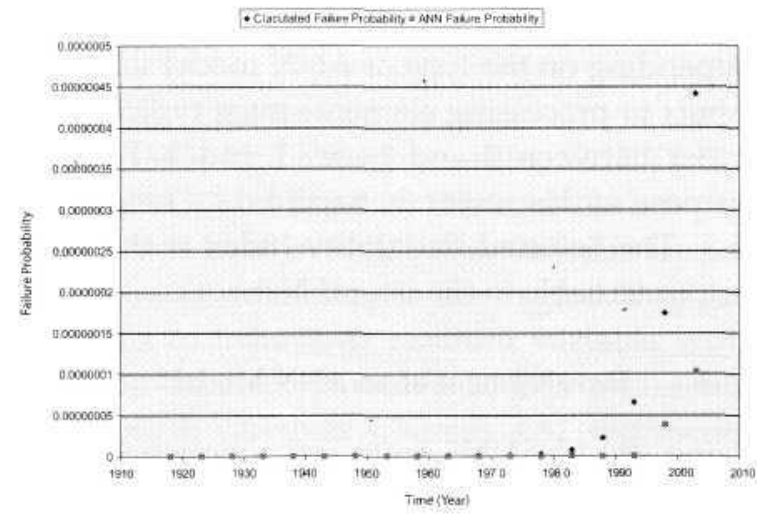

Figure 4 - Comparison of calculated and predicted Failure Probability for ANN training set

\section{Conclusion}

A back-propagation neural network was used to demonstrate the feasibility of ANNs to predict the service life of the masonry arch bridges. The results indicate that back-propagation neural networks have the capability of predicting the reliability index and estimating the failure probability and the service lives of masonry arch bridges with good accuracy.

Here, Reliability index was selected as basic parameter with related to service life of arch bridges. Based on that, it demonstrates the feasibility of ANN application forestimating the remaining service life of the structure.

Finally, knowing the failure probabilities of the selected arch bridge, the remaining service life of the structure can be estimated based on the estimated value from ANN.

\section{Acknowledgement}

The authors would like to give their sincere acknowledgement to the National Science Foundation of Sri Lanka for the financial support given through the research grant number RG/2003/E/001 and NSF/SCH/2005/ 04. They also highly appreciating the support given by the Road development Authority (RDA) in this regard.

\section{References}

1. Stamatios. V. Kartalopoulos, Understanding Neural Networks and Fuzzy logic, IEEE Inc. New York, 1996, Chapter 1, 2, \& 3.

2. James A. Anderson, an Introduction to Neural Network, MIT press, 1995.

3. James, A. Freeman., \& David, M. Skapura., Neural Networks Algorithms, Applycation, and Programming techniques, Addison-Wesley Publishing Company. Inc., New York, 1992, pp 141 \& pp 89-124.

4. Karunananda P.A.K. et. al., Service Life Prediction of Bridges using Structural Reliability Theory (M. Phil. Thesis) Department of Civil Engineering, Faulty of Engineering, University of Peradeniya, Sri Lanka, December 2004.

5. Dissanayake P.B.R et. al., Condition Evaluations of Bridges in National Road Network of Sri Lanka, Proceedings of Annual Research Session, University of Peradeniya, November 2004.

6. Karunananda P.A.K. et. al., Prediction of Serviceability Condition of Masonry Arch Bridges using Structural Reliability Theory, Proceedings of Annual Sri Lanka Association of Advancement of Science (SLAAS) research session, Colombo, Sri Lanka, 2004. 